\title{
EVOLUTIONARY MODELS FOR Be X-RAY BINARIES
}

C. de Loore and C. H. B. Sybesma

Astrophysical Institute

Free University Brussels, Belgium

Be $X$-ray binaries have the following general characteristics of interest for evolutionary scenarios: the spectral type of the non-compact star is B2 - 09 (e), the orbital periods are large, ranging from 16 to 581 days, the orbital eccentricities can be large, which could be related to the fact that the $\mathrm{X}$-ray stages are transient; the masses of the optical components range from 10 to $20 \mathrm{M}$ and their radii are relatively small $\left(5-10 \mathrm{R}_{\odot}\right)$. The optical luminosity is lower than $3 \times 10^{4} \mathrm{~L}_{\odot}$.

From these characteristics Rappaport and Van den Heuvel (1982) concluded that the components 1 ie well inside their Roche lobes, and that consequently Roche lobe overflow is not responsible for the $\mathrm{X}$-ray luminosity observed.

The aim of this paper is to make an analysis of possible sequences of massive binary evolution calculated with various types of massive binary evolution calculated with various assumptions, and to select plausible scenarios.

a) A first possible scenario is that of a 'lower' mass progenitor system $\left(10+8 \mathrm{M}_{\odot}\right)$ with an initial period of slightly more than 3 days. After evolution (without overshooting) and conservative mass transfer a white dwarf $+B$ star is obtained with a large period $(200 \mathrm{~d})$. Accretion onto the W.D. will trigger an explosion leading to the formation of a neutron star. The problem here is that accretion from the Be system has undergone RLOF. This is not supported by observational evidence;

b) A second approach is to start with higher mass stars (15-25 M $+8-15 \mathrm{M}_{0}$ ) and initial periods ranging from 5 to 7.75 days resulting in a case $B^{\odot}$ of mass transfer; after RLOF a He $+O B$ system is formed with periods in the range of 10-30 days. Therefore, even though there is period widening in the WR stage due to mass loss from stellar wind, further enlargement of the period as a result of the SN event is necessary.

c) When simultaneous, non-conservative evolution is calculated a good representation of the observed system becomes possible. A $15+10$ $\mathrm{M}_{0}\left(\mathrm{P}_{i}=8 \mathrm{~d}\right)$ system has as a pre-supernova system a $3.42+20.5 \mathrm{M}_{\mathrm{B}}$ system with a period of 68 days which is of the right order for a Be $X$-ray system (de Loore et al. 1985). 
d) Calculations of single stars show that overshooting from convective cores alters considerably the main-sequence evolution in the mass range involved here. Including overshooting it is possible to describe more accurately the wide main-sequence band between $\mathrm{M}_{i}-15 \mathrm{M}_{0}$ and $30 \mathrm{M}$. This may have a profound effect on the evolution of binaries $^{\Theta}$ (Doom 1985). Recalculation of the $15+10 \mathrm{M}$ system, including overshooting shows that this system goes through case A of mass transfer during which much less matter is transferred and as a result the final period is much smaller, and the mass of the accretion star remains rather low $\left(<12 \mathrm{M}_{\odot}\right)$. Calculations by Sybesma (1986) show that unless the initial mass ratio is close to 1 , leading to nonconservative mass exchange, case A of mass transfer will not lead to a Be X-ray binary progenitor system.

e) Calculation of a $20+18 \mathrm{M}$ system going through case $\mathrm{B}$ of mass transfer (initial periods: $20^{\circ}-40$ days) results in $W R+B$ systems with periods in the order of $40-78$ days with masses of $11.2+19 \mathrm{M}_{0}$, a promising pre-SN system eventually resulting in a $\mathrm{Be}$ $\mathrm{X}$-ray binary.

In order to obtain the very high required periods, as well as the high eccentricities observed in these systems an asymmetric SN explosion has to be invoked. This is in agreement with recent theoretical work by Arnett (1986). We conclude that:

- primaries of 15-25 $\mathrm{M}_{\odot}$ with initial periods of 5-25 days and Schwarzschild cores,

- primaries of 15-25 $\mathrm{M}_{\odot}$ with initial periods of 20-40 days and overshooting cores,

can evolve into pre-supernova systems with periods of $10-20 \mathrm{M}_{\odot}$ and periods of 10-100 days.

After asymmetric supernova explosions, systems are produced which match the observational characteristics of the $\mathrm{Be} X-\mathrm{ray}$ binaries, i.e. large periods, high eccentricities.

\section{References}

Arnett, W. D., 1986, personal communication. Doom, C., 1985, Astron. Astrophys., 142, 143. de Loore, C., Giovanelli, F., van Dessel, E. L., Bartolini, C., Burger, M., Ferrari-Tonioli, M., Giangrande, A., Guarnieri, A., Hellings, P., Hensberge, H., Persi, P., Piccioni, A., van Diest, H.: 1985, Astron. Astrophys., $141,279$.

Sybesma, C. H. B., 1986, Astron. Astrophys., 159, 108. 